\title{
Multiple QTL influence the serum $L p(a)$ concentration: a genome-wide linkage screen in the PROCARDIS study
}

Simona Barlera ${ }^{\star, 1,10}$, Claudia Specchia ${ }^{1,2,10}$, Martin Farrall ${ }^{3}$, Benedetta D Chiodini ${ }^{1,4}$, Maria Grazia Franzosi ${ }^{1}$, Stephan Rust ${ }^{5}$, Fiona Green ${ }^{3}$, Enrico B Nicolis ${ }^{1}$, John Peden ${ }^{3}$, Gerd Assmann ${ }^{5}$, Rory Collins ${ }^{6}$, Anders Hamsten ${ }^{7}$, Gianni Tognoni ${ }^{1}$, Hugh Watkins ${ }^{8}$, on behalf of the PROCARDIS Consortium ${ }^{9}$

\footnotetext{
${ }^{1}$ Department of Cardiovascular Research, Istituto di Ricerche Farmacologiche 'Mario Negri', Milano, Italy; ${ }^{2}$ Department of Biomedical Sciences and Biotechnologies, University of Brescia, Brescia, Italy; ${ }^{3}$ Department of Cardiovascular Medicine, Wellcome Trust Centre for Human Genetics, University of Oxford, Oxford, UK; ${ }^{4}$ Division of Genetics and Molecular Medicine, King's College London School of Medicine, London, UK; ${ }^{5}$ Leibniz-Institut für Arterioskleroseforschung an der Universität Münster, Münster, Germany; ${ }^{6}$ Clinical Trials Service Unit and Epidemiological Studies Unit, Richard Doll Building, University of Oxford, Oxford, UK; ${ }^{7}$ Atherosclerosis Research Unit, King Gustaf V Research Institute, Department of Medicine Solna, Karolinska Institute, Stockholm, Sweden;

${ }^{8}$ Department of Cardiovascular Medicine, John Radcliffe Hospital, Oxford, UK
}

The serum concentration of lipoprotein $\mathrm{Lp}(\mathrm{a})$ is known to be highly heritable and associated with cardiovascular risk. A genome-wide variance component linkage analysis was performed to localise quantitative trait loci (QTLs) influencing $L p(a)$ levels in a large cohort collected in the PROCARDIS coronary heart disease study. Highly significant linkage was detected at the previously described LP(a) locus on chromosome 6q27 (LOD 108). Taking into account the effect of the locus detected on chromosome 6, a highly significant LOD score was detected on chromosome 13q22-31 (LOD 7.0). Another significant region of linkage was observed on chromosomes 11p14-15 (LOD 3.5). The significant peak at 13q22-31 shows an essential overlap with a locus modulating cholesterol in familial hypercholesterolemia. If the gene underlying these loci is the same, it will be a promising candidate target for manipulating LDLcholesterol and $L p(a)$. We also detected linkage at a previously identified locus influencing $L p(a)$ on chromosome 1q23 (LOD 1.5). Our findings provide new and confirmatory information about genomic regions involved in the quantitative variation of $L p(a)$ and serve as a basis for further studies of candidate genes in these regions.

European Journal of Human Genetics (2007) 15, 221-227. doi:10.1038/sj.ejhg.5201732; published online 29 November 2006

Keywords: QTL; variance component; linkage analysis; lipoprotein (a)

\footnotetext{
*Correspondence: Dr S Barlera, Department of Cardiovascular Research, Istituto di Ricerche Farmacologiche 'Mario Negri', via Eritrea 62, 20157 Milano, Italy. Tel: + 3902 39014558; Fax: + 3902 33200049;

E-mail: simo@marionegri.it

${ }^{9}$ www.procardis.org

${ }^{10}$ These two authors contributed equally to this work.

Received 1 June 2006; revised 2 October 2006; accepted 4 October 2006; published online 29 November 2006
}

Introduction

$\mathrm{Lp}(\mathrm{a})$ is a lipoprotein formed by the assembly of LDL particles and a carbohydrate-rich protein, apolipoprotein(a) (apo(a)), which has a high degree of structural homology with plasminogen.

The existence of a moderately strong association of $\mathrm{Lp}(\mathrm{a})$ levels with atherosclerosis and coronary heart disease 
(CHD), independent of the standard vascular risk factors, is now clearly established. ${ }^{1-3}$ A meta-analysis on $5436 \mathrm{CHD}$ cases observed over 10 years found that people with $\mathrm{Lp}(\mathrm{a})$ levels in the top third of baseline measurement are at $\approx 70 \%$ increased risk of CHD compared with those in the bottom third. ${ }^{4}$

Several hypotheses exist about the underlying mechanisms by which $\operatorname{Lp}(\mathrm{a})$ contributes to the pathogenesis of atherosclerosis. Recently, Tsimikas et $a l^{5}$ advanced the hypothesis that, in the setting of enhanced oxidative stress, the binding of proinflammatory oxidised phospholipids by Lp(a) may, in part, mediate the atherogenicity of Lp(a).

The distribution of $\operatorname{Lp}(\mathrm{a})$ levels has been found to vary greatly among populations. In African-Americans, levels of $\operatorname{Lp}(\mathrm{a})$ are significantly higher when compared to those of European ancestry. ${ }^{6}$ Levels of Lp(a) are highly heritable and almost entirely genetically controlled; several studies estimated the heritability of this trait $>0.75^{7-10}$ and one study $>0.9$. $^{8}$

The apolipoprotein(a) (LPA) locus on chromosome 6q26-27 seems to explain most of the trait's variance and almost all the genome-wide scans investigating the $\operatorname{Lp}(\mathrm{a})$ trait obtained a very significant result at this locus. ${ }^{6,8,10,11}$ According to Broeckel, ${ }^{8}$ in a genome-wide scan on 513 western European families aimed at identifying chromosomal regions linked to myocardial infarction (MI) and related risk factors, 73\% of the overall $\mathrm{Lp}(\mathrm{a})$ heritability is explained by the LPA locus. In that study, the region including the LPA locus obtained a LOD score of $26.99 .^{8}$ Similarly, in a genome-wide scan of three different ethnic populations (non-Hispanic whites, African Americans and Hispanics), the LPA locus showed the strongest evidence for linkage with LOD scores of 18.62, 14.27 and 12.97 respectively. ${ }^{6}$ Thirty-four 'size polymorphism' alleles have been resolved at this locus and the length of the apo(a) isoform is inversely correlated with $\mathrm{Lp}(\mathrm{a})$ plasma concentrations and the risk of CHD.

Nevertheless, other (unlinked) genes are highly likely to contribute to the variance of $\mathrm{Lp}$ (a) levels. Broeckel ${ }^{8}$ found a novel linkage on chromosome 1q23, which seemed to explain $16 \%$ of the variance in $\operatorname{Lp}(\mathrm{a})$, but at least two subsequent studies have failed to replicate that finding. ${ }^{6,11}$

Our aim was to perform, on a large cohort of European ancestry collected through the PROCARDIS coronary heart disease study, a genome-wide variance component linkage analysis to localise genomic regions influencing $\mathrm{Lp}(\mathrm{a})$ levels, taking into account the effect of the LPA locus on chromosome 6 .

\section{Methods}

\section{Research design}

The PROCARDIS study is a European collaborative project to map susceptibility genes for coronary artery disease
(CAD). Ascertainment criteria for PROCARDIS probands were MI or symptomatic acute coronary syndrome (SACS; on the assumption that the latter represents a similar pathological process), according to modified WHO diagnostic criteria, ${ }^{12,13}$ occurring before the age of 65 years. The study recruited 2036 families with multiple CAD siblings. A detailed description of inclusion criteria as well as of the main findings of the PROCARDIS study has been reported elsewhere. ${ }^{14}$ DNA was extracted from a $9 \mathrm{ml}$ EDTA-anticoagulated sample of frozen whole blood using a standard technique. Genotyping has been performed using the ABI MD10 (Applied Biosystems, Warrington, UK) set of microsatellite markers for genome-wide screening. ${ }^{15}$ Ethics committee approval was obtained and all participants gave written informed consent. Families were recruited in four European countries, namely Germany, Italy, Sweden and UK.

\section{Phenotyping}

All participants filled out a standardized questionnaire that focused on the patient's baseline characteristics, cardiovascular risk factors, lifestyle and a detailed list of medications. Several biochemical measurements, such as lipids, lipoprotein subclasses, fibrinogen, insulin, pro-insulin and HbA1c have been collected during the study. Lipids and lipoprotein subclasses were determined by an NMR technique (LipoScience, Raleigh, USA). ${ }^{16}$

Although the main purpose of the PROCARDIS project is the identification of new susceptibility genes for CAD, phenotypes relevant to cardiovascular disease have been examined in our study with the aim to detect associated QTLs. Serum $\operatorname{Lp}(\mathrm{a})$ serum concentration was analysed as a continuous quantitative trait in a QTL genome-wide linkage analysis. Lp(a) was measured by an immunoturbimetric assay on a Hitachi 917 Roche automated clinical chemistry analyzer. ${ }^{17}$ From the PROCARDIS database, we selected all informative sibships in which Lp(a) levels had been measured in multiple siblings. The sibships include individuals with and without a personal history of CAD as the purpose of this analysis is the identification of loci associated to a quantitative trait. Use of lipid-lowering treatment (statins, fibrates or omega 3 preparations) was determined by participants' responses to questions about regular medication.

\section{Genotyping}

For the QTL analysis of Lp(a) levels, 493 markers at a mean spacing of $7 \mathrm{cM}$ for each of the 22 autosomes have been analysed (MD10 set). Marker allele frequencies were computed from the original data and inter-marker distances were based on the Rutgers sex-averaged map. ${ }^{18}$ Following this initial genome screen, 40 additional markers on chromosomes 3, 11 and 17 were genotyped to supplement the recovery of identity-by-descent (IBD) information over that extracted by the MD10 marker set 
(as reported in a supplementary table). Genotypic data were checked before statistical analysis using RELPAIR ${ }^{19}$ to confirm relationships and by $\mathrm{PEDCHECK}^{20}$ for inheritance inconsistencies.

\section{Statistical methods}

Descriptive analysis Summary statistics of the sample under investigation were calculated. Univariate and multivariate linear regression analyses were performed to test for factors associated with levels of $\operatorname{Lp}(\mathrm{a})$, using SAS 8.02. Covariates (age, sex, body mass index, systolic and diastolic blood pressure, triglycerides, total cholesterol, diabetes, lipid-lowering treatment) were originally chosen according to their biological influence on the trait and to their significant associations in a preliminary univariate regression analysis $(P$-value $<0.05)$. Sex, age and lipid-lowering treatment, statistically significant in the multivariate model implemented in SOLAR, were included in the quantitative analysis and retained in the final linkage analysis. In a quantitative analysis of $\operatorname{Lp}(\mathrm{a})$, the proportion of the total variance attributable to covariates and the covariate-adjusted heritability were estimated using a procedure implemented by SOLAR. ${ }^{21}$

Variance component linkage analysis A variance component linkage analysis was performed as implemented by the SOLAR computer program. ${ }^{21}$ Briefly, the variance component approach consists of fitting a linear mixed model, which involves estimating the trait mean and three components of variance: (a) an additive monogenic component linked to the region of interest or locus-specific effect; (b) a 'polygenic' component that incorporates overall familial effects or residual additive genetic effects; and (c) an 'environmental' component that incorporates effects unique to the individual, that is covariate effects, and individual-specific random environmental factors. ${ }^{22}$ To test the null hypothesis of no linkage to a QTL, the likelihood of a model with the additive genetic variance due to the QTL equal to zero was compared with a model with the additive variance due to the QTL equal to that estimated by SOLAR. Lp(a) has been studied as a continuous variable and has been log-transformed (natural logarithm) to adjust for non-normality because the variance component approach is sensitive to certain distributional assumptions. ${ }^{23}$ The method requires an estimate of the expected genetic covariances between relatives as a function of the IBD sharing at a given chromosomal location. While SOLAR estimates multipoint IBD sharing probabilities, the exact IBD has been calculated using MERLIN ${ }^{24}$ and then imported into SOLAR. Genetic locations for the marker loci are scaled in Haldane centimorgan (cM). Multipoint variance component linkage analysis was carried out at constant increments of $1 \mathrm{cM}$ along all the autosomes.
Oligogenic linkage analysis A multipoint oligogenic linkage analysis to identify multiple loci affecting the variation in $\mathrm{Lp}(\mathrm{a})$ was carried out. $^{25}$ In this sequential strategy, the genome was repeatedly scanned for linkage and the chromosomal location that yielded the largest marginal LOD score was retained for further conditional analyses. The conditional modelling approach is analogous to a stepwise forward-selection procedure used in linear regression and other statistical analyses. Ab initio, a conventional single-locus variance-components analysis is used to identify the strongest linkage signal. At each subsequent step, QTLs identified in the previous step are fixed in location and the conditional oligogenic QTL method scans for further QTL. The QTL-specific effects at all loci included in the model are (re)estimated at each step. A stoppingcriterion of LOD scores 1.9 was used to terminate the stepwise procedure. ${ }^{26}$ Oligogenic analyses are expected to yield more accurate estimates of the relative effect of each locus and to increase the power to detect linkages. This approach allowed other promising regions of linkage to be highlighted after adjustment for the variance attributed to the LPA locus as well as to other linkage peaks.

Simulation analysis The pointwise (nominal) significance of the LOD scores was empirically assessed by simulation of the conditional linkage analysis process by using a procedure in SOLAR. ${ }^{21}$ A total of 10000 replicates of a fully informative marker, completely unlinked to the QTL influencing the trait, have been performed to obtain the empirical distribution of the LOD scores and to determine the pointwise $P$-value associated with the original LOD score. To estimate the significance level of the resulting LOD scores, taking into account the number of chromosomes scanned and the density of the markers, we calculated the genome-wide $P$-values using the Gaussian approximation described by Feingold. ${ }^{27}$

Regression-based linkage analysis To complement the variance components linkage analysis, a model-free regression method was applied which is generally robust to selection and distributional issues. ${ }^{28}$ This method regresses the estimated IBD sharing between non-inbred relative pairs on the squared sums and differences of their trait value. Lp(a) trait was log-transformed and then adjusted for sex, age and lipid-lowering treatment by reference to an external epidemiological sample (PROCAM project http://www. chd-taskforce.de/slidekits.htm). An ascertainment correction was implemented by specifying the trait-mean and variance in the unselected PROCAM cohort (mean $=13.7 \mathrm{mg} / \mathrm{dl}$ s.d. $=18.8 \mathrm{mg} / \mathrm{dl}$ ) in the MERLIN-REGRESS analysis.

\section{Results}

Pedigree data extracted from the PROCARDIS database consisted of 1812 families with 4012 individuals genotyped 
and checked for inheritance inconsistencies, having data available for the serum $\operatorname{Lp}(\mathrm{a})$ concentration and for the covariates retained in the analysis. Table 1 summarizes the phenotypic characteristics of the individuals included in the study.

Lp(a) levels ranged from 1 to $361 \mathrm{mg} / \mathrm{dl}$ with a mean value of $52.7 \mathrm{mg} / \mathrm{dl}$ and a standard deviation of $50.1 \mathrm{mg} / \mathrm{dl}$, whereas the median value was $31.0(\mathrm{IQR}=14.5-82.0) \mathrm{mg} / \mathrm{dl}$. Values of skewness and kurtosis for $\operatorname{Lp}(\mathrm{a})$ were 1.4 and 2.0 and decreased respectively to -0.4 and -0.1 for the logtransformed $\operatorname{Lp}(\mathrm{a})$ that was used for the linkage scan. The following statistically significant covariates have been used for adjustment of the $\operatorname{Lp}(\mathrm{a})$ trait: sex, age and lipidlowering treatment. In order to show how Lp(a) levels vary with sex, age and treatment, parameter estimates and standard errors obtained from the multivariate quantitative regression analysis are reported in Table 2 . The polygenic heritability for $\mathrm{Lp}(\mathrm{a})$ was estimated by SOLAR to be $0.91 \pm 0.03$, whereas the proportion of variance owing to covariates was equal to 0.01 .

Table 1 Phenotypic characteristics of the 4012 individuals

\begin{tabular}{lc}
\hline Characteristics & Frequency (\%) or Mean \pm SD \\
\hline No. of families & 1812 \\
No. of individuals & 4012 \\
Sex & \\
$\quad$ Males & $2690(67 \%)$ \\
$\quad$ Females & $1322(33 \%)$ \\
No of individuals with CAD & $3195(80 \%)$ \\
Age (years) & $61.6 \pm 8.3$ \\
Lipid-lowering treatment & $2182(54 \%)$ \\
Diabetics & $527(13 \%)$ \\
Smokers & $408(10 \%)$ \\
Lp(a) (mg/dl) & $52.9 \pm 49.9^{*}$ \\
Body mass index $\left(\mathrm{kg}^{*} \mathrm{~m}^{2}\right)$ & $28.2 \pm 4.4^{*}$ \\
Total cholesterol $\left(\mathrm{mg} \mathrm{dl}^{-1}\right)$ & $180.4 \pm 36.9^{*}$ \\
LDL cholesterol $\left(\mathrm{mg} \mathrm{dl}^{-1}\right)$ & $113.1 \pm 31.1^{*}$ \\
HDL cholesterol $\left(\mathrm{mg} \mathrm{dl}^{-1}\right)$ & $45.2 \pm 12.5^{*}$ \\
Triglycerides $\left(\mathrm{mg} \mathrm{dl}^{-1}\right)$ & $144.6 \pm 78.1^{*}$ \\
Systolic BP $\left(\mathrm{mmHg}^{*}\right)$ & $140.5 \pm 22.3^{*}$ \\
Diastolic BP $\left(\mathrm{mmHg}^{*}\right)$ & $82.8 \pm 12.0^{*}$ \\
\hline
\end{tabular}

* Mean and SD (standard deviation) are calculated by fitting a regression model where familiar cluster has been taken into consideration.

Table 2 Parameter estimates and Standard Errors (SE) from the multivariate quantitative regression analysis of logarithm of $L p(a)$ implemented by SOLAR

\begin{tabular}{lrcc}
\hline Covariates & Estimate & SE & P-value \\
\hline Age (years in continuous) & 0.022 & 0.009 & 0.025 \\
Sex (females) & 0.693 & 0.161 & $<0.0001$ \\
Lipid lowering treatment & -0.671 & 0.152 & $<0.0001$ \\
(not treated) & & & \\
\hline
\end{tabular}

Multipoint oligogenic linkage analysis to identify multiple loci affecting the variation in $\operatorname{Lp}(\mathrm{a})$ was carried out. As expected the highest LOD score in the initial genome scan was found on chromosome 6 at the same location as the LPA locus, which is known to be positioned in the region $6 \mathrm{q} 26-\mathrm{q} 27$ at a physical distance of $161 \mathrm{Mb}$ (Figure 1 ). The maximum LOD score observed in our analysis was 108.3 at $189 \mathrm{cM}$ (location expressed using Haldane cM) flanked by markers D6S1581 and D6S1599 at a physical distance of $160.2-162.8 \mathrm{Mb}$. The total additive genetic variance explained by this locus was $0.74 \pm 0.03$. After adjusting for the effects of the locus detected on chromosome 6, a high LOD score was detected on chromosome 13 between D13S156 and D13S265 (LOD 7.0, maximum at D13S170). In the third step of the conditional linkage procedure, a significant linkage peak was found on chromosome 11 (LOD 3.5) between markers D11S902 and D11S904. Further regions of tentative linkage were observed on chromosome 15 (LOD 2.9) between D15S131 and D15S205 and on chromosome 19 (LOD 2.7) between D19S571 and D19S418. We also observed a LOD score equal to 1.5 at $170 \mathrm{cM}$, flanked by markers D1S498 and D1S484 (148.1-157.6 Mb), close to a new locus influencing $\mathrm{Lp}(\mathrm{a})$ levels identified at marker D1S1679 $(159.1 \mathrm{Mb})$ by Broeckel. ${ }^{8}$ LOD plots of the main findings are reported in Figure 2 with maximum LOD score values highlighted.

To assess the significance level of our linkage results we estimated the empirical $P$-values by using a simulation of 10000 replicates. Pointwise $P$-values as well as locus specific heritabilities are reported in Table 3. Furthermore, the Gaussian approximation proposed by Feingold ${ }^{27}$ suggests these linkages attain genome-wide significance on chromosome $13(P<0.0001)$ and on chromosome 11

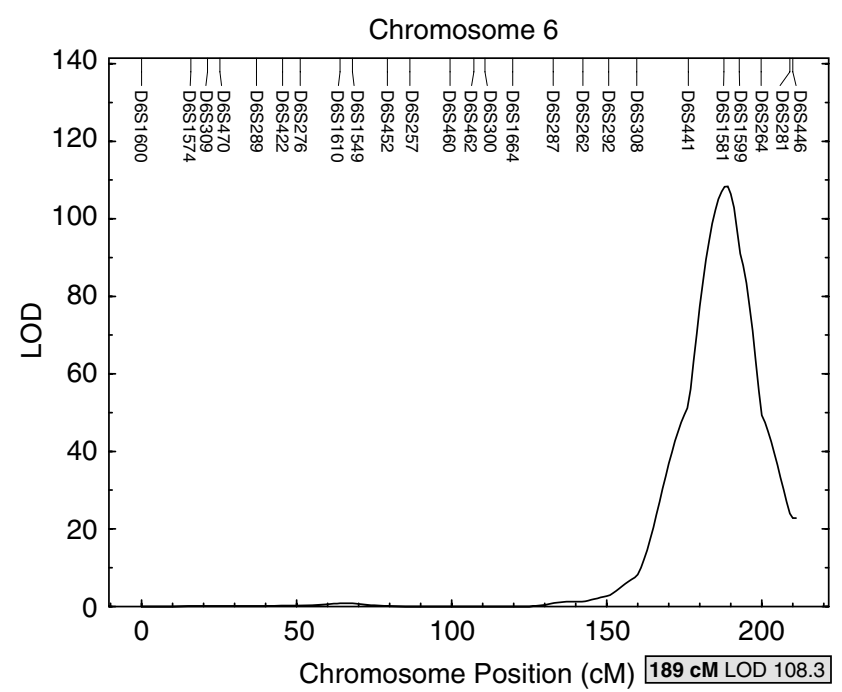

Figure 1 Oligogenic linkage analysis: multipoint LOD plot for logarithm of $\mathrm{Lp}(\mathrm{a})$ on chromosome 6 (location expressed using Haldane $\mathrm{CM}$ ). 

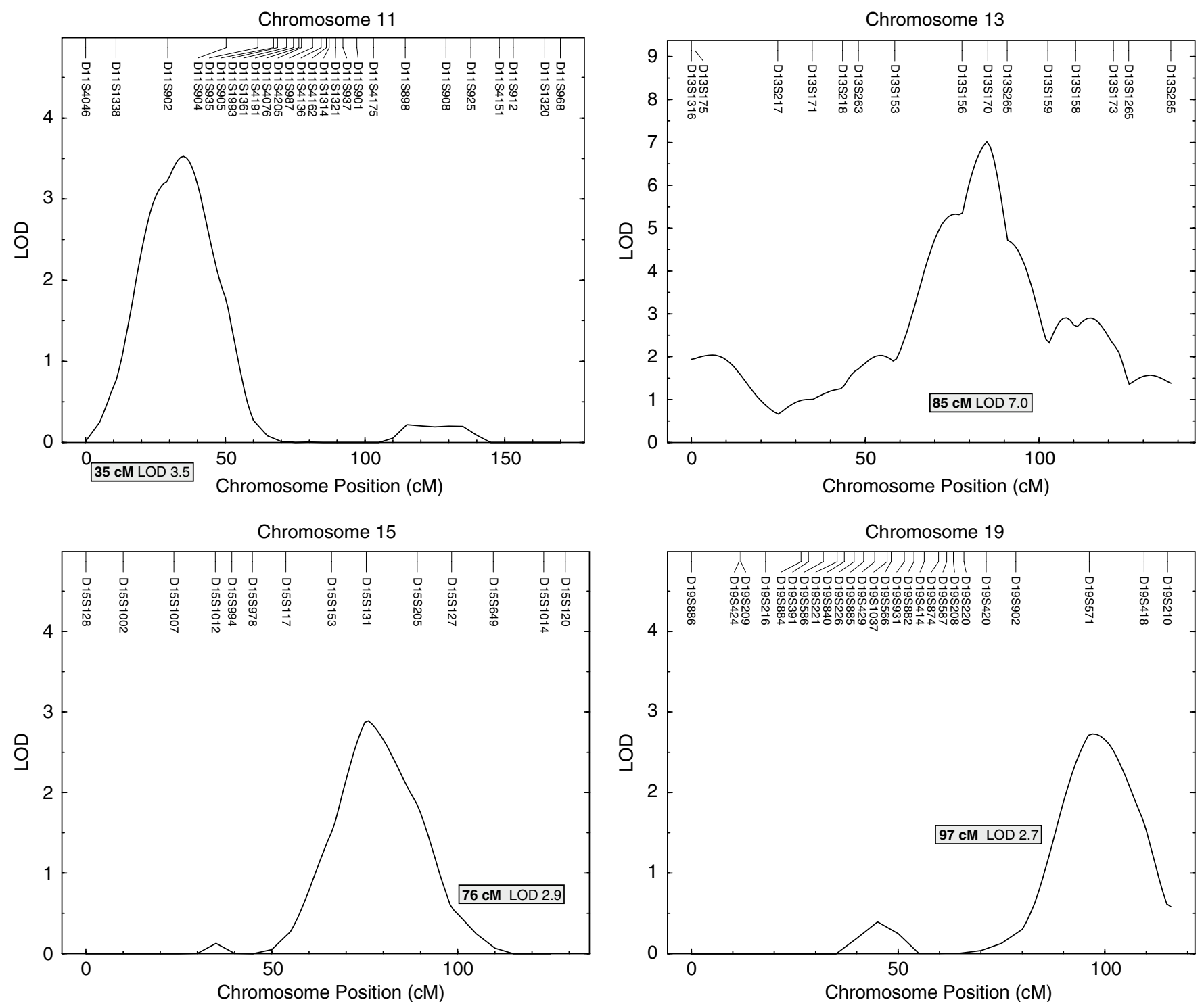

Figure 2 Oligogenic linkage analysis: multipoint LOD plots for logarithm of $L p(a)$ on chromosomes 11, 13, 15 and 19. Maximum LOD scores $(\geq 1.9)$ are reported (location expressed using Haldane cM).

Table 3 Results of the oligogenic analysis of variation in Lp(a) levels. Physical location, LOD score with associated empirical pointwise $P$-value and locus specific heritability $(\mathrm{H} 2)$

\begin{tabular}{lcccr}
\hline Chromosome & Flanking markers & Physical location (Mb) & Sequential LOD score & Empirical P-value \\
\hline 11 & D11S902 - D11S904 & $17.4-26.6$ & 3.5 & 0.0007 \\
13 & D13S156 - D13S265 & $73.5-89.2$ & 7.0 & 0.0001 \\
15 & D15S131 - D15S205 & $68.9-82.0$ & 2.9 & 0.08 \\
19 & D19S571 - D19S418 & $57.9-60.2$ & 2.7 & 0.003 \\
\hline
\end{tabular}

$(P=0.0139)$. Regression-based approach implemented by MERLIN-REGRESS detected QTL on chromosome 6 $(\mathrm{LOD}=43.4)$ and chromosome $13(\mathrm{LOD}=1.9)$.

\section{Discussion}

Complex traits such as serum $\operatorname{Lp}(\mathrm{a})$ are known to be influenced by environmental and genetic factor. We have 
performed a genome-wide screen on a large cohort of informative families to search for genetic components influencing $\mathrm{Lp}$ (a) levels. As expected, we found a high heritability of the trait of about $91 \%$ which supports the existence of a strong genetic component also taking into account environmental covariates.

A highly significant linkage was detected on chromosome 6 at 189 cM (LOD 108.3) flanked by markers D6S1581 and D6S1599 (160.2-162.8 Mb) at the same location as the apolipoprotein(a) gene, which is on chromosome 6q26-27 at a physical location of $161 \mathrm{Mb}$ (map positions according to http://genome.ucsc.edu/, May 2004).

Then, we extended the analysis to an oligogenic model taking into account, in the sequential scanning of the genome, the effect of the locus detected on chromosome 6 and of other major loci detected in our analysis. We have estimated genome-wide significance thresholds that are appropriate for this linkage experiment with a Gaussian approximation. Significant conditional linkage was found on chromosome 13q22-31 as well as on chromosome 11p15. Two further tentative linkages were identified on chromosomes 15q23-25 and 19q13.4.

Interestingly, the 13q22.1-13q31.1 locus between D13S156 and D13S265 (73.5-89.2 Mb) found in our genome-wide linkage analysis for $\mathrm{Lp}(\mathrm{a})$ overlaps a region that modulates LDL cholesterol in patients with familial hypercholesterolemia mapped to D13S156-D13S158 $(73.5-102.8 \mathrm{Mb})$, as reported by Knoblauch ${ }^{29}$ and further supported by analysis of healthy twins that produced a maximum LOD score at marker D13S1241 $(96.3 \mathrm{Mb})$ for a QTL influencing LDL-cholesterol. ${ }^{29}$ In PROCARDIS, pretreatment LDL cholesterol values were not available and the mean cholesterol was lower than in the general population rendering any modulating genetic effects inaccessible (Table 1). In contrast, mean $\operatorname{Lp}(\mathrm{a})$ is higher in PROCARDIS and thus the effect of genetic modulation remains visible. It may turn out that the linkage results at this locus are due to the same underlying gene, which could be a candidate target for manipulating LDL-cholesterol and $\operatorname{Lp}(\mathrm{a})$.

A study by Barkley ${ }^{6}$ also reported strong signals on chromosome 19 at 30 and $47 \mathrm{cM}$ (Kosambi), in a clearly different position from that obtained in our study at $97 \mathrm{cM}$ (Haldane). However, as a confirmatory result, we found a LOD score equal to 1.5 between markers D1S498 and D1S484 (148.1-157.6 Mb), approximately at the same location as the new locus identified by Broeckel $^{8}$ and colleagues on chromosome 1q23 at marker D1S1679 $(159.1 \mathrm{Mb})$ on a sample of 513 families for a total of 1406 individuals $(\mathrm{LOD}=3.81)$. It is reasonable to consider our result as evidence for replication of this finding. Interestingly, the population in Broeckel's study was also ascertained on the basis of pre-mature coronary heart disease.

The population here investigated was, in fact, previously selected on the basis of a CAD diagnosis, as the primary aim of the PROCARDIS study was the investigation of susceptibility genes for CAD. Eighty percent of the individuals analysed were affected by CAD and Lp(a) levels in our sample are quite high when compared to those of the general population. ${ }^{30}$ Although ascertainment bias may not bias the 'evidence for linkage', it might decrease the power to detect genetic effects, because it tends to decrease the correlation among relatives and increases the rate of Type II error. ${ }^{31}$ The ascertainment correction implemented in SOLAR is designed for extended pedigrees recruited through a single proband with an extreme quantitative phenotype. Ascertainment in our affected sib pairs depends on clinical phenotypes in at least two sibs and only indirectly on quantitative intermediate traits such as Lp(a). Consequently, it was not possible to make an appropriate adjustment for ascertainment in the SOLAR analysis. However, an alternative regression-based linkage approach (MERLIN-REGRESS) has been devised to detect linkage robustly in selected samples. By applying this method, epidemiological information regarding the distribution of $\mathrm{Lp}$ (a) levels in an unselected population (PROCAM) was incorporated into the linkage analysis. The MERLIN-REGRESS analysis confirmed the same locations on chromosome 6 and 13, although the LOD scores were considerably smaller. The regression-based linkage analysis takes into account the ascertainment issue, but it does not implement the oligogenic (conditional) analysis model (which can be fitted in SOLAR). Our results suggest that important loci can emerge in an oligogenic analysis that are not apparent in single-locus analyses; a finding that is consistent with experiences of applying multivariate regression models in general statistical analysis. We conclude that each analysis can provide complementary insights into QTL architecture and that further methodological developments are needed to efficiently analyse human linkage data with complex ascertainment.

Our findings provide new and important information about genomic regions involved in the quantitative variation of $\operatorname{Lp}(\mathrm{a})$. The lack of consistency with loci linked in some previous studies might suggest that the genetic components of the $\operatorname{Lp}(\mathrm{a})$ trait are complex to detect, involving many genes with different effects. Moreover our study population, involving a total of more than 4000 individuals, the majority affected by CAD and having different environmental backgrounds, may differ from those of previous similar genome-scans.

Finally, these results provide further insights into the oligogenic control of circulating $\mathrm{Lp}(\mathrm{a})$ levels that will encourage candidate gene studies that aim to determine the molecular basis of the underlying QTL.

\section{Acknowledgements}

We are grateful to the families for their generosity in participating in the study and to the numerous medical and technical staff who assisted in this project. The study was initiated under a European 
Union funded programme and thereafter received funding from AstraZeneca $A B ; H W$ and $R C$ also acknowledge support from the British Heart Foundation and RC from the Medical Research Council, AH obtained support for this project from the Swedish Heart-Lung Foundation, the Swedish Medical Research Council (8691), the Torsten and Ragnar Söderberg Foundation, the Petrus and Augusta Hedlund Foundation, the Karolinska Institute and the Stockholm County Council. we thank Laura Almasy for extensive technical advice on SOLAR and Marcella Devoto for her helpful suggestions.

The full membership of the PROCARDIS consortium is listed at www.procardis.org.

\section{References}

1 Nguyen TT, Ellefson RD, Hodge DO, Bailey KR, Kottke TE, AbuLebdeh HS: Predictive value of electrophoretically detected lipoprotein(a) for coronary heart disease and cerebrovascular disease in a community-based cohort of 9936 men and women. Circulation 1997; 96: 1390-1397.

2 Wild SH, Fortmann SP, Marcovina SM: A prospective casecontrol study of lipoprotein(a) levels and apo(a) size and risk of coronary heart disease in Stanford Five-City Project participants. Arteriosclerosis, Thromb Vasc Biol 1997; 17: 239-245.

3 Berg K, Dahlen G, Christophersen B, Cook T, Kjekshus J, Pedersen $\mathrm{T}$ : Lp(a) lipoprotein level predicts survival and major corollary events in the Scandinavian Simvastatin Survival Study. Clin Genet 1997; 52: 254-261.

4 Danesh J, Collins R, Peto R: Lipoprotein(a) and coronary heart disease. Meta-analysis of prospective studies. Circulation 2000; 102: $1082-1085$.

5 Tsimikas S, Brilakis ES, Miller ER et al: Oxidized phospholipids, Lp(a) lipoprotein, and coronary artery disease. N Engl J Med 2005; 353: 46-57.

6 Barkley RA, Brown AC, Hanis CL, Kardia SL, Turner ST, Boerwinkle E: Lack of genetic linkage evidence for a trans-acting factor having a large effect on plasma lipoprotein[a] levels in African Americans. J Lipid Res 2003; 44: 1301-1305.

7 Boerwinkle E, Leffert CC, Lin J, Lackner C, Chiesa G, Hobbs HH: Apolipoprotein(a) gene accounts for greater than $90 \%$ of the variation in plasma lipoprotein(a) concentrations. J Clin Invest 1992; 90: 52-60.

8 Broeckel U, Hengstenberg C, Mayer B et al: A comprehensive linkage analysis for myocardial infarction and its related risk factors. Nat Genet 2002; 30: 210-214.

9 Austin MA, Sandholzer C, Selby JV, Newman B, Krauss RM, Utermann G: Lipoprotein(a) in women twins: heritability and relationship to apolipoprotein(a) phenotypes. Am J Hum Gene 1992; 51: 829-840.

10 Ober C, Abney M, McPeek MS: The genetic dissection of complex traits in a founder population. [erratum appears in Am J Hum Genet 2002 Jan;70(1):284]. Am J Hum Gene 2001; 69: 1068-1079.

11 Beekman M, Heijmans BT, Martin NG et al: Two-locus linkage analysis applied to putative quantitative trait loci for lipoprotein(a) levels. Twin Res 2003; 6: 322-324.

12 WHO: Nomenclature and criteria for diagnosis of ischemic heart disease. Report of the Joint International Society and Federation of Cardiology/World Health Organization task force on standardization of clinical nomenclature. Circulation 1979; 59: 607-609.
13 Gillum RF, Fortmann SP, Prineas RJ, Kottke TE: International diagnostic criteria for acute myocardial infarction and acute stroke. Am Heart J 1984; 108: 150-158.

14 Farrall M, Green FR, Peden JF et al: Genome-wide mapping of susceptibility to coronary artery disease identifies a novel replicated locus on chromosome 17. PLOS Gene 2006; 2: e72.

15 Dechairo B, Dimon C, van Heel D et al: Replication and extension studies of inflammatory bowel disease susceptibility regions confirm linkage to chromosome 6p (IBD3). Eur J Hum Gene 2001; 9: 627-633.

16 Otvos JD: Measurement of lipoprotein subclass profiles by nuclear magnetic resonance spectroscopy. Clini Lab 2002; 48: $171-180$.

17 Siekmeier R, Marz W, Scharnagl H et al: Determination of lipoprotein(a): Comparison of a novel latex enhanced immunoturbidimetric assay and a immunoradiometric assay [In:German]. Lab Medizin 1996; 20: 294-298.

18 Kong X, Murphy K, Raj T, He C, White PS, Matise TC: A combined linkage-physical map of the human genome. [erratum appears in Am J Hum Genet. 2005 Feb;76(2):373]. Am J Hum Gene 2004; 75: $1143-1148$.

19 Boehnke M, Cox NJ: Accurate inference of relationships in sibpair linkage studies. Am J Hum Gene 1997; 61: 423-429.

20 O'Connell JR, Weeks DE: PedCheck: A program for identification of genotype incompatibilities in linkage analysis. Am J Hum Gene 1998; 63: 259-266.

21 Almasy L, Blangero J: Multipoint quantitative-trait linkage analysis in general pedigrees. Am J Hum Gene 1998; 62: 1198-1211, May.

22 Amos CI: Robust variance-components approach for assessing genetic linkage in pedigrees. Am J Hum Gene 1994; 54: 535-543.

23 Allison DB, Neale MC, Zannolli R, Schork NJ, Amos CI, Blangero $\mathrm{J}$ : Testing the robustness of the likelihood-ratio test in a variancecomponent quantitative-trait loci-mapping procedure. Am J Hum Gene 1999; 65: 531-544.

24 Abecasis GR, Cherny SS, Cookson WO, Cardon LR: Merlin - rapid analysis of dense genetic maps using sparse gene flow trees. [see comment]. Nat Genet 2002; 30: 97-101.

25 Blangero J, Almasy L: Multipoint oligogenic linkage analysis of quantitative traits. Genetic Epidemiology 1997; 14: 959-964.

26 Lander E, Kruglyak L: Genetic dissection of complex traits: guidelines for interpreting and reporting linkage results. Nature Genetics 1995; 11: 241-247.

27 Feingold E, Brown PO, Siegmund D: Gaussian models for genetic linkage analysis using complete high-resolution maps of identity by descent. American Journal of Human Genetics 1993; 53: 234-251.

28 Sham PC, Purcell S, Cherny SS, Abecasis GR: Powerful regressionbased quantitative-trait linkage analysis of general pedigrees. [see comment]. American Journal of Human Genetics 2002; 71: $238-253$.

29 Knoblauch H, Muller-Myhsok B, Busjahn A et al: A cholesterollowering gene maps to chromosome 13q. American Journal of Human Genetics 2000; 66: 157-166.

30 Falchi M, Andrew T, Snieder H, Swaminathan R, Surdulescu G, Spector T: Identification of QTLs for serum lipid levels in a female sib-pair cohort: a novel application to improve the power of two-locus linkage analysis. Human Molecular Genetics 2005; 14: 2971-2979.

31 Comuzzie AG, Williams JT: Correcting for ascertainment bias in the COGA data set. Genetic Epidemiology 1999; 17: S109-S114. 